\title{
NETWORK CODING-AWARE RATE CONTROL AND SCHEDULING IN WIRELESS NETWORKS
}

\author{
Hulya Seferoglu ${ }^{\dagger}$, Athina Markopoulou ${ }^{\dagger}$, Ulas Kozat ${ }^{\ddagger}$ \\ ${ }^{\dagger}$ University of California, Irvine and ${ }^{\ddagger}$ Docomo USA Labs
}

\begin{abstract}
Network coding has been recently applied to wireless networks to increase throughput. It is typically implemented as a thin layer between MAC and IP, transparently to higher layers. In this paper, we study rate control and scheduling over wireless networks with intersession network coding, as a utility maximization problem. We demonstrate that there is benefit from making rate control and scheduling aware of the underlying network coding. The key intuition is that network coding introduces new network coded flows and eventually new conflicts between nodes, which should be taken into account both in rate control and in scheduling. We compare the network coding-aware to the network coding-unaware schemes in two cases: (i) when optimal control is used and (ii) when practical, suboptimal control is used. Our main goal is to make the case for network coding-aware rate control and scheduling, via simulation of representative examples. Along the way, we also propose a practical scheme that approximates the optimal control.
\end{abstract}

Index Terms - Network coding, wireless networks, rate allocation, scheduling, resource allocation, utility maximization, cross-layer design.

\section{INTRODUCTION}

Recently, it has been shown that network coding (i.e. mixing packets from different flows at wireless routers, so as to increase the information content of each transmission) combined with broadcast transmission over wireless, can increase the overall throughput $[1,2]$. In this scenario, network coding is typically implemented as a thin layer between MAC and IP, transparently to higher layers. For example, in [2], standard TCP has been used on top of a multi-hop wireless network with one-hop opportunistic network coding (COPE [2]). In this paper, we focus on rate control and scheduling over such networks, and we argue that it is important to do a cross-layer optimization, i.e., make end-to-end rate control and scheduling aware of the underlying network coding operations. We define what network coding "awareness" means and we demonstrate its benefits over network coding "unaware" - both optimal and suboptimal (practical) - schemes.

Our key intuition is the following. When network coding is used, the achievable rate region gets extended by coding some flows together and broadcasting them. However, this introduces additional conflicts. For example, when two flows transmitted in reverse directions are coded together at an intermediate node, there is a new network coded flow created, which may conflict with other flows transmitted in the neighborhood. In this paper, we argue that this information should be explicitly taken into account by higher layers, namely rate control and scheduling, in order to fully exploit the extended achievable rate region.

There is a large body of work on resource allocation problems in communication networks in general, in wireless networks in particular $[3,4]$, and recently also considering network coding [5-7]. This paper builds on our recent work in [8]. There, we formulated the problem of rate allocation and scheduling over wireless networks with one-hop opportunistic network coding (as in COPE [2]) as a utility maximization problem showed that it can be decomposed into two independent problems (rate-control and scheduling) and developed a distributed solution for the rate control part, especially for video. In this paper, we first compare the optimal network coding-aware rate control and scheduling scheme of [8] to the corresponding optimal network coding-unaware scheme; we demonstrate the benefit of network coding awareness via simulation in an illustrative example. Second, we design a suboptimal but practical NC-aware scheme by (i) converting the flow-based to a packet-based rate control, (ii) employing efficient heuristics for scheduling as in [9], and (iii) proposing a scheme for signaling the information among nodes. We then compare the performance (achievable rate-region) of the practical NC-aware scheme to that of the corresponding NCunaware practical scheme.

The rate control schemes studied in this paper provide insights into the interaction of network coding and rate control and can eventually be used as a guideline to make practical media transport protocols, such as TFRC, network codingaware. The structure of the rest of the paper is as follows. Section 2 gives an overview of the system. Section 3 compares the optimal NC-aware and NC-unaware schemes. Section 4 presents and compares practical, suboptimal versions of the previous optimal schemes. Section 5 concludes the paper.

\section{SYSTEM OVERVIEW}

Wireless Network. We consider a wireless mesh network with $N$ nodes. Nodes are connected to other nodes in the same communication range through hyperarcs. A hyperarc $(i, J)$ is a collection of links from node $i$ to a non-empty set of nodes $J \subset N$, which are in the range of $i$ and are interested in receiving the same network code through a broadcast transmission from $i$. Let $A$ be the set of all hyperarcs. The network can be viewed as a directed hypergraph $H=$ 


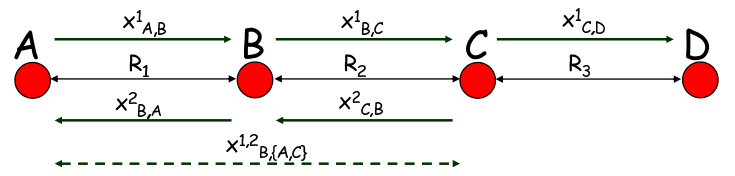

Fig. 1. Motivating example. A sends flow $x_{1}$ to $D$ through nodes $A$ and B. C sends flow $x_{2}$ to A through B. (Node B codes together parts of flows $x_{1}$ and $x_{2}$ into a new network coded flow $x_{B,\{A, C\}}^{1,2}$ and broadcasts it to both A and $\mathrm{C}$. This network coded flow conflicts with the transmission of uncoded flow $x_{C, D}^{1}$ from $\mathrm{C}$ to $\mathrm{D}$.)

$(N, A)$. Transmissions over different hyperarcs may interfere with each other. We consider the protocol model of interference [10], according to which, each node can either transmit or receive at the same time and all transmissions in the range of the receiver are considered as interfering hyperarcs. For a hypergraph $H$, the conflict graph $A_{c}$ is defined to have nodes that correspond to hyperarcs and edges that indicate interference between them. A clique $A_{c}^{q}$ in the conflict graph consists of several hyperarcs, at most one of which can transmit at the same time without interference.

Sources/Flows. Let $S$ be the set of flows transmitted over this network, between some known source-destination pairs. Each flow sends at rate $x_{s}$ and has utility function $U_{s}\left(x_{s}\right)$, which is an increasing and concave function of $x_{s}$.

Routing. In this paper, we consider that each flow $s$ follows a single path $I_{s}$ from the source to the destination. Paths are pre-determined by a routing protocol and given as input to our problem. However, note that several hyper-arcs may connect two consecutive nodes in the path, as explained below.

Network coding. Intermediate nodes use one-hop opportunistic network coding. They mix packets from different flows, using simple network coding operations (XOR) as in COPE [2] and broadcast the coded packets to the one-hop neighborhood. Nodes in the same neighborhood listen to each other's transmission and store overheard packets; these are used later to decode received coded packets and also to construct new coded packets. We focus on the scenario where network coding opportunities are created by traffic patterns of cross-flows and by overhearing packets. For example, such cross-patterns are created by the chain, " $X$ ", cross, and wheel topologies in [2].

Rate control and Scheduling. Assuming that the content of the codes is constructed by the underlying COPE scheme, and we focus on rate control (i.e., determining the rates that should be assigned to every source and every code) and scheduling (i.e., the nodes that transmit and the percentage of time that each hyperarc should be used for a code).

Example. The example shown in Fig.1 demonstrates the key intuition why we need network coding "awareness". There are two flows in reverse directions: node $\mathrm{A}$ transmits Flow-1 with rate $x_{1}$ to node $\mathrm{D}$ via nodes $\mathrm{B}$ and $\mathrm{C}$; node $\mathrm{C}$ transmits Flow-2 with rate $x_{2}$ to node A via node B. All nodes transmit in the same channel and at the same power level. The link capacities $\left(R_{1}, R_{2}, R_{3}\right.$ for links $\left.\mathrm{AB}, \mathrm{BC}, \mathrm{CD}\right)$ inversely depend on the distance between the node pairs.

Let $x_{A, B}^{1}, x_{B, C}^{1}$, and $x_{C, D}^{1}$ be the uncoded parts of Flow1 , while $x_{C, B}^{2}$ and $x_{B, A}^{2}$ are the uncoded parts of Flow-2.
$x_{B,\{A, C\}}^{1,2}$ is the network coded part of Flow-1 and Flow-2: node $\mathrm{B}$ combines parts of $x_{1}$ and $x_{2}$ and broadcasts to both $\mathrm{A}$ and C. From the flow conservation we have that: $x_{1}=$ $x_{A, B}^{1}=x_{B, C}^{1}+x_{B,\{A, C\}}^{1,2}=x_{C, D}^{1}$ and $x_{2}=x_{C, B}^{2}=$ $x_{B, A}^{2}+x_{B,\{A, C\}}^{1,2}$. If the scheme is $N C$-aware, it will take into account that there is a new network coded flow, transmitted with rate $x_{B,\{A, C\}}^{1,2}$ and interfering with all other transmissions in the system. Therefore, no simultaneous transmissions will take place. However, if the scheme is $N C$-unaware, it does not know that there is a transmission (or hyperarc) for the network coded flow from B to A and C. Therefore, when link $\{B, A\}$ is used for transmission of Flow-2 and network coding is possible with packets of Flow-1, then packets are combined and transmitted. However, since transmissions from $\mathrm{B}$ to $\mathrm{A}$ and $\mathrm{C}$ to $\mathrm{D}$ are considered as interference-free, in the NC-unaware scheme, they will be scheduled at the same time. Thus network coding at $\mathrm{B}$ may lead to a collision at $\mathrm{C}$ when scheduling decisions are done in a NC-unaware fashion. As a result, network coding opportunities can be wasted and the total achieved flow rate in the worst case reduces to the scenario where no network coding is applied.

\section{OPTIMAL RATE CONTROL AND SCHEDULING}

Optimal NC-Aware Scheme: In [8], we followed the linkbased approach for cross-layer optimization of wireless networks $[3,4]$ and extended it to include network coding. The goal is to optimize the total utility:

$$
\begin{array}{ll}
\max _{x, \tau} & \sum_{s \in S} U_{s}\left(x_{s}\right) \\
s . t & H_{i, J}^{s} x_{i, J}^{s} \leq R_{i, J} \tau_{i, J}^{k} \xi_{i, J}^{s}, \forall k \in K, \forall s \in S_{k}, \forall(i, J) \in A \\
& x_{s}=\sum_{\left\{J \mid(i, J) \in A, i \in I_{s}\right\}} x_{i, J}^{s}, \forall s \in S, i \in I_{s} \\
& \sum_{(i, J) \in A_{c}^{q}} \sum_{k \in K} \tau_{i, J}^{k} \leq \gamma, \forall A_{c}^{q} \in A_{c} \\
& x_{s} \geq 0, \forall s \in S, x_{i, J}^{s} \geq 0, \forall s \in S, \forall(i, J) \in A, \\
& \tau_{i, J}^{k} \geq 0, \forall(i, J) \in A, \forall k \in K
\end{array}
$$

The rate control aims at selecting the rate $x_{s} \geq 0$ at each source $s \in S$, and the parts of it $\left(x_{i, J}^{s} \geq 0\right)$ that are transmitted over each hyperarc $\{i, J\}$ on the path $I_{s}$ using predetermined network codes $k \in K$, where $K$ is the set of network codes over $i, J$. The first constraint refers to flows $\left(s \in S_{k}\right.$ ) coded together in the same network code $k$ and transmitted over the hyperarc $(i, J)$ if the indicator function $\left(H_{i, j}^{s}\right)$ shows that flow $s$ is transmitted over hyperarc $(i, J)$ (i.e. $H_{i, J}^{s}=1$ ). These flows coexist and do not compete for the total rate $R_{i, J} \tau_{i, J}^{k} \xi_{i, J}^{s}$ allocated to code $k$, where $R_{i, J}$ is the maximum achievable rate over hyperarc $(i, J), \tau_{i, J}^{k}$ is the percentage of time that hyperarc $(i, J)$ is used for network code $k \in K$, and $\xi_{i, J}^{s}$ is the probability of successful transmission from node $i$ to its destination node $j \in J$. Thus, $R_{i, J} \tau_{i, J}^{k} \xi_{i, J}^{s}$ is the effective rate used by code $k$ after excluding packet losses. The maximum rate of these network coded 
flows ( $s \in S_{k}$ ) should be up to the effective rate of the code $k$, which means that the rate of each one of them $\left(H_{i, j}^{s} x_{i, J}^{s}\right)$ should be up to $R_{i, J} \tau_{i, J}^{k} \xi_{i, J}^{s}$. The second constraint is the flow conservation of flow $x_{s}$ at each hop on its path $I_{s}$ towards the destination. Note that a flow may be transmitted over different hyperarcs with different network codes and rates $x_{i, J}^{s}$ which are summed up to $x_{s}$ with this constraint. The third constraint captures the conflicts due to interference, similarly to [6]: different network codes over the same hyperarcs and nodes in the same clique cannot transmit at the same time. They share the available transmission time, or equivalently the rate where $\gamma$ is an over-provisioning factor, in practice less than 1 to account for channel access and collisions.

The first set of constraints can be relaxed with Lagrangian multipliers $q_{i, J}^{k, s}$ and the problem can be decomposed into a separate rate control and a scheduling part. Due to lack of space, we refer the interested reader to [8] for details. In particular, the rate control problem can be further decomposed into a number of rate-control subproblems, each of which can be solved independently at each source using only feedback from the network about the queue sizes $q_{i, J}^{\eta_{i, J}(s), s}$. We note that, at each node, we maintain one queue per network code; this information is taken into account by each source $s$ when controlling $x_{s}$ and $x_{i, J}$. The scheduling problem must be solved for all the hyperarcs in the network considering the interference model, so as to determine the percentage of time $\tau_{i, J}^{k}$ that hyperarc $\{i, J\}$ should be used for network code $k$. This problem is known to be NP-hard [3]. Finally, the parameters are updated iteratively using a subgradient method: $q_{i, J}^{k, s}(t+1)=\left\{q_{i, J}^{k, s}(t)+\beta_{t}\left[H_{i, J}^{s} x_{i, J}^{s}-R_{i, J} \tau_{i, J}^{k} \xi_{i, J}^{s}\right]\right\}^{+}$.

Optimal NC-Unaware Scheme: To quantify the benefit of network coding awareness, we compare the optimal NC-aware scheme to the corresponding optimal NC-unaware scheme. The latter scheme takes decisions based on (i) the total queue length at each link of every node and (ii) the conflict graph; however, it does not know the more detailed information about the queue size per coded or uncoded flow. In the example of Fig. 1, there are actually 3 queues (one for uncoded flow $x_{B, C}^{1}$, one for uncoded flow $x_{B, A}^{2}$, and one for the coded flow $x_{B,\{A, C\}}^{1,2}$ ), which are known to the NC-aware scheme. However, the NC-unaware scheme only knows the existence of two output queues: one for link BA and one for link BC. Apart from being agnostic to the existence of network coding, the optimal NC-unaware scheme is similar to the NC-aware one: it can be formulated using the link-centric approach [3],

$$
\begin{aligned}
\max _{x, \tau} & \sum_{s \in S} U_{s}\left(x_{s}\right) \\
\text { s.t } & \sum_{s \in S} H_{i, j}^{s} x^{s} \leq R_{i, j} \tau_{i, j} \xi_{i, j}, \forall(i, j) \in A \\
& \sum_{(i, j) \in A_{c}^{q}} \tau_{i, J} \leq \gamma, \forall A_{c}^{q} \in A_{c} \\
& x_{s} \geq 0, \forall s \in S, \tau_{i, j} \geq 0, \forall(i, j) \in A,
\end{aligned}
$$

and can also be solved using Lagrangian decomposition. In the rate control part, each source simply determines its rate
Table 1. Optimal Schemes. Achievable rates for the example of Fig. 1 for four different scenarios: Scenario 1: $R_{1}=1, R_{2}=1, R_{3}=1$; Scenario 2: $R_{1}=1, R_{2}=2, R_{3}=1$; Scenario 3: $R_{1}=1, R_{2}=2, R_{3}=2$; Scenario 4: $R_{1}=1, R_{2}=4, R_{3}=1$.

\begin{tabular}{|c||c|c|c||c|c|c|}
\hline \multicolumn{1}{|c||}{} & \multicolumn{3}{c|}{ Optimal NC-aware } & \multicolumn{3}{c|}{ Optimal NC-unaware } \\
\hline \hline Scenarios & $x_{1}$ & $x_{2}$ & $x_{1}+x_{2}$ & $x_{1}$ & $x_{2}$ & $x_{1}+x_{2}$ \\
\hline 1 & 0.21 & 0.39 & 0.60 & 0.20 & 0.40 & 0.60 \\
2 & 0.25 & 0.50 & 0.75 & 0.33 & 0.32 & 0.65 \\
3 & 0.31 & 0.46 & 0.77 & 0.32 & 0.30 & 0.62 \\
4 & 0.33 & 0.50 & 0.83 & 0.38 & 0.37 & 0.75 \\
\hline
\end{tabular}

$x_{s}$. The scheduling problem is solved considering all conflicting links in the system. Each queue is updated when packets are transmitted or received, without distinguishing whether they are delivered with or without network coding.

Optimal NC-Aware vs. Optimal NC-Unaware Scheme. In Table 1, we present numerical results of the achieved rates for the example of Fig. 1 for four different scenarios, which correspond to different values of link rates $R_{1}, R_{2}, R_{3}$ for links $A B, B C, C D$, respectively. The results are generated with 500 iterations. The utility function is assumed to be $U_{s}\left(x_{s}\right)=\log \left(x_{s}\right)$. The optimal solution is obtained using the Lagrangian decomposition outlined in this section.

We can see that, in all scenarios, the NC-aware scheme achieves the same or higher total rate $\left(x_{1}+x_{2}\right)$ compared to the NC-unaware scheme. In some scenarios, the NC awareness brings significant improvement: this usually happens in scenarios where (i) the underlying conflicts of network coded flows reduce the network coding opportunities and (ii) there are no network opportunities over different links.

\section{PRACTICAL RATE CONTROL \& SCHEDULING}

We now propose a practical implementation of the optimal NC-aware and NC-unaware rate control and scheduling schemes. We use simulations to show that they are good approximations of the optimal schemes and we also compare them to quantify the benefit from NC awareness in practice.

To create a practical scheme we need to make the following modifications: (i) implement a packet-based rate control that approximates the optimal flow-based rate control (ii) implement a low-complexity yet efficient heuristic that approximates the optimal scheduling (iii) signal information about queue size and conflicts (iv) construct and update queues. These modifications apply similarly to both the NC-aware and NC-unaware schemes with some minor differences, as described below.

Rate Control. The optimal NC-aware rate control determines the flow rate of each network coded flow according to the queue length of each network code on every node on the flow's path. Instead, we use the following heuristic, using an approach similar to [9], to perform rate control at each packet. Each source maintains an average rate value for each of its network coded rates. At each transmission opportunity (iteration in the optimal solution) the optimal rates are compared to the average rates. If the average rate of a network coded part of a flow is less than its optimal value, a packet is inserted to the transmission buffer and labeled with the network coding 
policies for each node on its path; a packet is coded according to its label at each node on its path to the destination. The NC-unaware rate control employs exactly the same scheme but maintains the average rate at each source instead of the average rates for all coded flows.

Scheduling. Scheduling determines the nodes and flows that will transmit and the percentage of time a node will transmit. Because this problem is NP-hard [3], we use a heuristic similar to [9]. Since a node can conflict with at most two hop neighbors, the optimal scheduling can be found by considering only one and two hop conflicts as follows. Each node maintains information about the queue size of its own flows and of its neighbors' flows and exchanges this information with its neighbors at the end of each packet transmission. This way, every node learns the queue sizes of its one- and two-hop neighbors, and compares its own queue sizes (for each network coded flow) with the queue sizes of these neighbors. If a node has the largest queue, it tries to transmit a packet from this queue, by selecting a small initial value for the contention window in 802.11. This is a good approximation of the optimal solution due to its low complexity and the simplicity of information exchange. The practical NC-unaware scheduling uses the same mechanism.

Signaling. Queue size information is exchanged after transmitting a packet. In practice, when 802.11 is used as an underlying MAC mechanism, the queue size information can be exchanged via RTS/CTS control packets, as in [9]. In addition to the queue sizes, we consider that queue destination information is also appended to the control packets, in order for each node to determine its exposed terminals. ${ }^{1}$ In summary, at each RTS/CTS transmission, nodes exchange their queue sizes, destination nodes and their neighbors' queue sizes and destination nodes. In addition, queue size information at each node over the path of a source is passed to the source via feedback. The signaling mechanism of the practical NC-unaware scheme is exactly the same.

Parameter Update. In the NC-aware case, each node maintains a queue for each source and network code, which gets updated when a packet is transmitted or received. In the NC-unaware case, information is maintained for each output queue (link) at each node. However, even when two or more packets are transmitted from/to a queue or more than one queue transmits due to network coding, the queues are updated accordingly.

Simulation Results. We now present simulation results for the practical $\mathrm{NC}$-aware and $\mathrm{NC}$-unaware schemes for the example of Fig.1. Table 2 shows the rates achieved by the practical schemes in the same four scenarios discussed in section 3. There are two observations to make from this table. First and more important, the NC-aware scheme achieves higher total rate than the NC-unaware one, in this practical case as well and for all four scenarios. Second, comparing the practical schemes to the corresponding optimal schemes,

\footnotetext{
${ }^{1}$ We consider that two nodes whose destination nodes are in different interference regions can transmit at the same time even if they are interfering to each other (exposed terminal problem) by figuring out conflicts considering destination nodes. We consider a synchronization among nodes to eliminate collisions of ACKs and control packets.
}

Table 2. Practical Schemes. Achievable rates for the example of Fig. 1 for four different scenarios: Scenario $1: R_{1}=1, R_{2}=1, R_{3}=1$; Scenario 2: $R_{1}=1, R_{2}=2, R_{3}=1$; Scenario 3: $R_{1}=1, R_{2}=2, R_{3}=2$; Scenario 4: $R_{1}=1, R_{2}=4, R_{3}=1$.

\begin{tabular}{|c||c|c|c||c|c|c|}
\hline \multicolumn{1}{|c||}{} & \multicolumn{3}{c|}{ Practical NC-aware } & \multicolumn{3}{c|}{ Practical NC-unaware } \\
\hline \hline Scenarios & $x_{1}$ & $x_{2}$ & $x_{1}+x_{2}$ & $x_{1}$ & $x_{2}$ & $x_{1}+x_{2}$ \\
\hline 1 & 0.19 & 0.36 & 0.55 & 0.19 & 0.35 & 0.54 \\
2 & 0.24 & 0.46 & 0.70 & 0.29 & 0.30 & 0.59 \\
3 & 0.28 & 0.44 & 0.72 & 0.29 & 0.30 & 0.59 \\
4 & 0.28 & 0.46 & 0.74 & 0.35 & 0.37 & 0.72 \\
\hline
\end{tabular}

we see that the rates achieved are lower. This is expected as the practical schemes are suboptimal: e.g. the control packets (RTS/CTS) use $10 \%$ of transmission time and reduce the total rate. Third, the rates achieved by the practical schemes are close to the optimal rates, which indicates that they are efficient heuristics. Finally, we checked the convergence of the practical NC-aware and NC-unaware schemes in this example and found that all rates and queue sizes converged to their operating point; we omit the figures due to lack of space.

\section{CONCLUSION}

In this paper, we considered wireless networks with one-hop opportunistic network coding and we argued in favor of making rate control and scheduling network-coding aware. We gave the main intuition and also compared NC-aware vs. NCunaware, both optimal and practical, schemes. In addition to the formulations, we presented simulation results for an illustrative example with two cross flows, which captures the main intuition and should be a building block of any large scenario with cross-flows and network coding. In general, the benefit from the NC awareness depends on (i) the network coding opportunities (ii) the conflicts of network coded with other flows and (iii) the link rates/capacities where (i) and (ii) depend on the topology and traffic scenario. An exhaustive characterization of a wide range of scenarios is part of future work.

\section{REFERENCES}

[1] Y. Wu, P.A. Chou, and S. Y. Kung, "Information exchange in wireless network coding and physical layer broadcast," in Proc. of IEEE CISS, Baltimore, MD, March 2005.

[2] S. Katti, H. Rahul, W. Hu, D. Katabi, M. Medard, and J. Crowcroft, "XORs in the air: Practical network coding," in Proc. of ACM SIGCOMM 2006

[3] X. Lin, N. B. Schroff, R. Srikant, "A tutorial on cross-layer optimization in wireless networks," in IEEE Journal on Selected Areas in Communication, vol. 24(8), pp. 1452-1463, Aug. 2006.

[4] X. Lin, N. B. Shroff, "Joint rate control and scheduling in multihop wireless networks", in Proc. of Decision and Control, vol. 2, pp. 14841489, Dec. 2004.

[5] P. Chaporkar, A. Proutiere, "Adaptive network coding and scheduling for maximizing througput in wireless networks," in Proc. of ACM Mobicom, Montreal, Canada, Sep. 2007.

[6] S. Sengupta, S, Rayanchu, S. Banarjee, "An Analysis of Wireless Network Coding for Unicast Sessions: The Case for Coding-Aware Routing," in Proc. of Infocom, 2007

[7] T. Cui, L. Chen, T. Ho, "Energy Efficient Opportunistic Network Coding for Wireless Networks," in Proc. of Infocom, 2008.

[8] H.Seferoglu, A.Markopoulou, "Distributed Rate Control for Video Streaming over Wireless Networks with Intersession Network Coding", to appear in Proc. of Packet Video 2009.

[9] U. Akyol, M. Andrews, p. Gupta, J. Hobby, I. Saniee, A. Stolyar, "Joint scheduling and congestion control in mobile ad-hoc networks," in Proc. of Infocom, 2008.

[10] P. Gupta, P. R. Kumar, "The Capacity of Wireless Network," in IEEE Trans. on Inf. Theory, vol. 34(5), pp. 910-917, 2000 